\title{
Using Geographic Information Systems (GIS) to Identify Communities in Need of Health Insurance Outreach: An OCHIN Practice-based Research Network (PBRN) Report
}

\author{
Heather Angier, MPH, Sonja Likumahuwa, MID, MPH, Sean Finnegan, MS, \\ Trisha Vakarcs, Christine Nelson, PhD, RN, Andrew Bazemore, MD, MPH, \\ Mark Carrozza, MA, and Jennifer E. DeVoe, MD, DPhil
}

Background: Our practice-based research network (PBRN) is conducting an outreach intervention to increase health insurance coverage for patients seen in the network. To assist with outreach site selection, we sought an understandable way to use electronic health record (EHR) data to locate uninsured patients.

Methods: Health insurance information was displayed within a web-based mapping platform to demonstrate the feasibility of using geographic information systems (GIS) to visualize EHR data. This study used EHR data from 52 clinics in the OCHIN PBRN. We included cross-sectional coverage data for patients aged 0 to 64 years with at least 1 visit to a study clinic during $2011(n=228,284)$.

Results: Our PBRN was successful in using GIS to identify intervention sites. Through use of the maps, we found geographic variation in insurance rates of patients seeking care in OCHIN PBRN clinics. Insurance rates also varied by age: The percentage of adults without insurance ranged from $13.2 \%$ to 86.8\%; rates of children lacking insurance ranged from $1.1 \%$ to $71.7 \%$. GIS also showed some areas of households with median incomes that had low insurance rates.

Discussion: EHR data can be imported into a web-based GIS mapping tool to visualize patient information. Using EHR data, we were able to observe smaller areas than could be seen using only publicly available data. Using this information, we identified appropriate OCHIN PBRN clinics for dissemination of an EHR-based insurance outreach intervention. GIS could also be used by clinics to visualize other patient-level characteristics to target clinic outreach efforts or interventions. ( $\mathrm{J}$ Am Board Fam Med 2014;27:804-810.)

Keywords: Electronic Medical Records, Geographic Information Systems, Health Insurance, Practice-based Research

The Affordable Care Act (ACA) has provided new opportunities for individuals to obtain health insurance, ${ }^{1}$ yet many could still experience gaps in coverage because of switching between different pro-

This article was externally peer reviewed.

Submitted 17 January 2014; revised 1 April 2014; accepted 4 April 2014.

From the Department of Family Medicine, Oregon Health \& Science University, Portland (HA, SL, JED); the Robert Graham Center, Washington, DC (SF, AB); OCHIN, Inc., Portland, OR (TV, CN, JED); and the Health Foundation of Greater Cincinnati, Cincinnati, OH (MC).

Funding: This work was financially supported by the Agency for Health care Research and Quality (AHRQ) (grant no. 1 R01 HS018569); the Patient-Centered Outcomes Research Institute (PCORI), PFA Cycle I Contract grams. ${ }^{2}$ Health insurance facilitates access to health care services and improves outcomes; ${ }^{3-5}$ therefore these gaps are concerning. Electronic health record (EHR) functions used to manage chronic disease ${ }^{6,7}$ have been shown to be effective in tracking patients' health insurance coverage, ${ }^{8}$ and panel man-

(2012) and PCORI, CDRN (2014), Health Systems; and the Oregon Health \& Science University Department of Family Medicine.

Conflict of interest: JED is an editorial board member of the $7 A B F M$.

Corresponding author: Heather Angier, MPH, Department of Family Medicine, Oregon Health \& Science University, 3181 SW Sam Jackson Park Road, Mailcode: FM, Portland, OR 97239 (E-mail: angierh@ohsu.edu). 
agement systems could be adapted to identify patients and reach out to those without insurance. 9 Practice-based research networks (PBRNs) are an ideal setting in which to develop and test the effectiveness of tools to monitor patients' insurance status $^{8}$ and to facilitate communications to patients about new health insurance programs. ${ }^{10}$

To investigate ways to address this important issue, our PBRN developed and is currently testing tools to identify patients without health insurance and conduct outreach to assist them in gaining appropriate coverage. ${ }^{10}$ The OCHIN PBRN is made up largely of community health centers (CHCs) that have historically cared for patients regardless of health insurance status. Thus, many of the patients at these CHCs will benefit from new ACA health insurance programs. ${ }^{1,11} \mathrm{We}$ worked with our PBRN steering committee and patient engagement panel (PEP) on this project. The PBRN steering committee includes a diverse group of stakeholders with varying degrees of research experience (eg, clinicians, practice managers, academic researchers), and the PEP is made up of patients who are seen in network clinics. To decide which clinics should take part in the study to test the tools, the committee wanted to know which clinics saw the largest percentages of uninsured patients. Tabular data could have been used to highlight the population attributes of the network, but we have found that complicated data tables are difficult to digest, especially for stakeholders without research expertise. Therefore, we wanted to test the feasibility of using geographic information systems (GIS) as an alternative way of presenting EHR data to make understanding easier.

GIS technologies have shown promise in helping public health officials and health care systems to better understand their communities' and patients' health and to geographically visualize factors that affect health. ${ }^{12,13}$ For example, GIS has been used to show neighborhood assets and needs, ${ }^{14,15}$ map disease clusters, ${ }^{16-18}$ and plan the location of health care services. ${ }^{19-22}$ Few studies have experimented with the use of GIS to visualize EHR data, ${ }^{12,23}$ and to our knowledge, EHR data has not been previously used to visualize health insurance coverage for a population of patients based on their geographic location. Thus, in this article we demonstrate the use of GIS to visualize patients' coverage status from EHR data as a way to engage stakeholders in identifying potential intervention sites to target for a research study.

\section{Methods}

\section{Study Setting and Data Sources}

OCHIN, Inc. (originally called the Oregon Community Health Information Network, renamed "OCHIN" as other states joined), is a collaboration of CHCs and other primary care organizations that serve vulnerable populations. ${ }^{24}$ OCHIN provides and maintains a comprehensive EHR infrastructure for all member clinics, including practice management data (similar to insurance claims data), and medical record data. The data are checked, cleaned, and stored in an electronically searchable central repository. ${ }^{25}$ All OCHIN clinics share this single, linked EHR, and patients have one unique record across all sites. The OCHIN PBRN, founded in 2006, includes all OCHIN members, representing more than 300 clinics across 18 states. $^{24}$

\section{Study Population and Variables}

This study used EHR data from 52 OCHIN clinics in Oregon that had implemented full EHR capabilities by January 1, 2011. Oregon OCHIN clinics are located throughout the state in both urban and rural areas and reflect the population of the state; the western portion of Oregon is the most populous area and includes the largest cities (i.e., Portland, Eugene, and Salem). All patients aged $<1$ to 64 years with at least 1 visit to 1 of these clinics between January 1, 2011, and December 31, 2011, were included in the study $(\mathrm{N}=228,284)$. We excluded patients $>64$ years of age because most 65 -year-old patients are eligible for Medicare and less likely to lack insurance.

Insurance status was assessed cross-sectionally at each patient's first visit during the study year (2011). We categorized the patients' insurance status as not insured, privately insured, or publicly (including Medicaid and/or Medicare) insured at that 1 visit only. We stratified results by age because Medicaid insurance eligibility rules are different for individuals $<19$ years old. We aggregated patients by their insurance status within ZIP codes. ZIP codes that contained $<10$ patients in any of the insurance categories were excluded.

\section{Spatial Visualization}

This study was conducted in collaboration with The Robert Graham Center and The Health Foundation of Greater Cincinnati, who are leaders in using GIS mapping tools for health innovation 
and community needs assessment. Several of their data portals integrate data from diverse sources such as the Centers for Disease Control and Prevention, the National Center for Health Statistics, the US Census Bureau, and statewide Medicaid and Medicare data.

We extracted relevant study data from the OCHIN EHR into a de-identified data set that contained aggregated counts of patients by ZIP code. Patient health insurance information was displayed within the HealthLandscape, LLC, mapping platform, which combines and displays various sources of health, socioeconomic, and environmental information to characterize communities and promote better understanding of their health. ${ }^{26}$ We engaged the PBRN steering committee and the $\mathrm{PEP}$ in an iterative process to create communityrelevant displays for web-based mapping of insurance coverage. OCHIN PBRN members used the mapping tool to identify practices with large numbers of patients without health insurance coverage. This study was approved by the institutional review board at our institution.

\section{Results}

Our PBRN was successful in using GIS to help identify intervention sites. Through the use of maps, we found geographic variation in insurance rates of patients seeking care in OCHIN PBRN clinics. Insurance rates also varied by age: fewer adults than children had coverage. The percentage of adults without insurance ranged from a low of only $13.2 \%$ seen in 1 practice to a high of $86.8 \%$. The percentage of children ( $<19$ years old) without insurance coverage ranged from $1.1 \%$ to $71.7 \%$. With few exceptions, geographic locations with higher rates of adults without insurance also had higher rates of children lacking insurance (Figure 1).

OCHIN clinics serving the highest percentage of patients without insurance were located in 4 counties in the state of Oregon: Lincoln, Benton, Polk, and Marion (Figure 2). We used GIS to compare this information to other communitylevel characteristics to see insurance rates in conjunction with other social determinants of health. For example, there were some areas with the lowest median household income that also had low insurance rates, though not all areas followed this pattern (Figure 2). For example, a large percentage of
OCHIN CHC patients in Benton County were not insured, but median incomes were near the national average, ranging from $\$ 48,600$ to $\$ 60,658$ per year.

\section{Discussion}

This article demonstrates how EHR data can be deployed in a web-based GIS mapping tool and used by stakeholders to identify areas in need of a specific intervention. The study identified ZIP codes with the highest percentage of uninsured patients. We used these findings, along with population density information and input from the steering committee and the PEP on the needs of the potential communities, to identify sites to disseminate EHR-based health insurance outreach tools. These tools, designed by the OCHIN PBRN and clinic end users, were created to support outreach to patients who may be eligible for health insurance programs and are currently being pilot tested. ${ }^{10}$ More information regarding this project will become available once the tools are implemented throughout the clinics and their utility studied (Figure 3).

In the future, we envision GIS being used for research and by clinics in our PBRN. GIS could be used for OCHIN PBRN projects, including recruitment of practices into studies aligned with practice and community needs and dissemination and implementation strategies to target the densest geographic areas. For example, the tools designed to conduct health insurance outreach could later be used to affect those who are underinsured; our PBRN could use GIS to visualize and identify the areas with large percentages of patients without appropriate coverage. We found that EHR data offer benefits beyond the types of data usually available in GIS maps, such as enabling visualization of a smaller area than might be possible with publicly available data (eg, US Census data). Using EHR data can also help to identify areas where publicly available data might not be sufficient for understanding the characteristics of a community. In our case, EHR data were helpful to see patients living in a median income area (based on publicly available data) with large numbers of patients without insurance (based on EHR data). With this finding, we were able to identify clinics that might not have been targeted for an intervention if we used publicly available data alone, without the EHR data.

In addition to assisting with project decisions, the OCHIN PBRN is exploring how to make GIS 
Figure 1. Children and Adults without Health Insurance: Rates Visualized from Electronic Health Record Data from Clinics in the OCHIN Practice-based Research Network (PBRN), by ZIP code. (A) Percentage of children without insurance at first visit: <1-18 years of age with at least one visit to any OCHIN PBRN clinic in 2011. (B) Percentage of adults without insurance at first visit: 19-64 years of age with at least one visit to any OCHIN PBRN clinic in 2011.
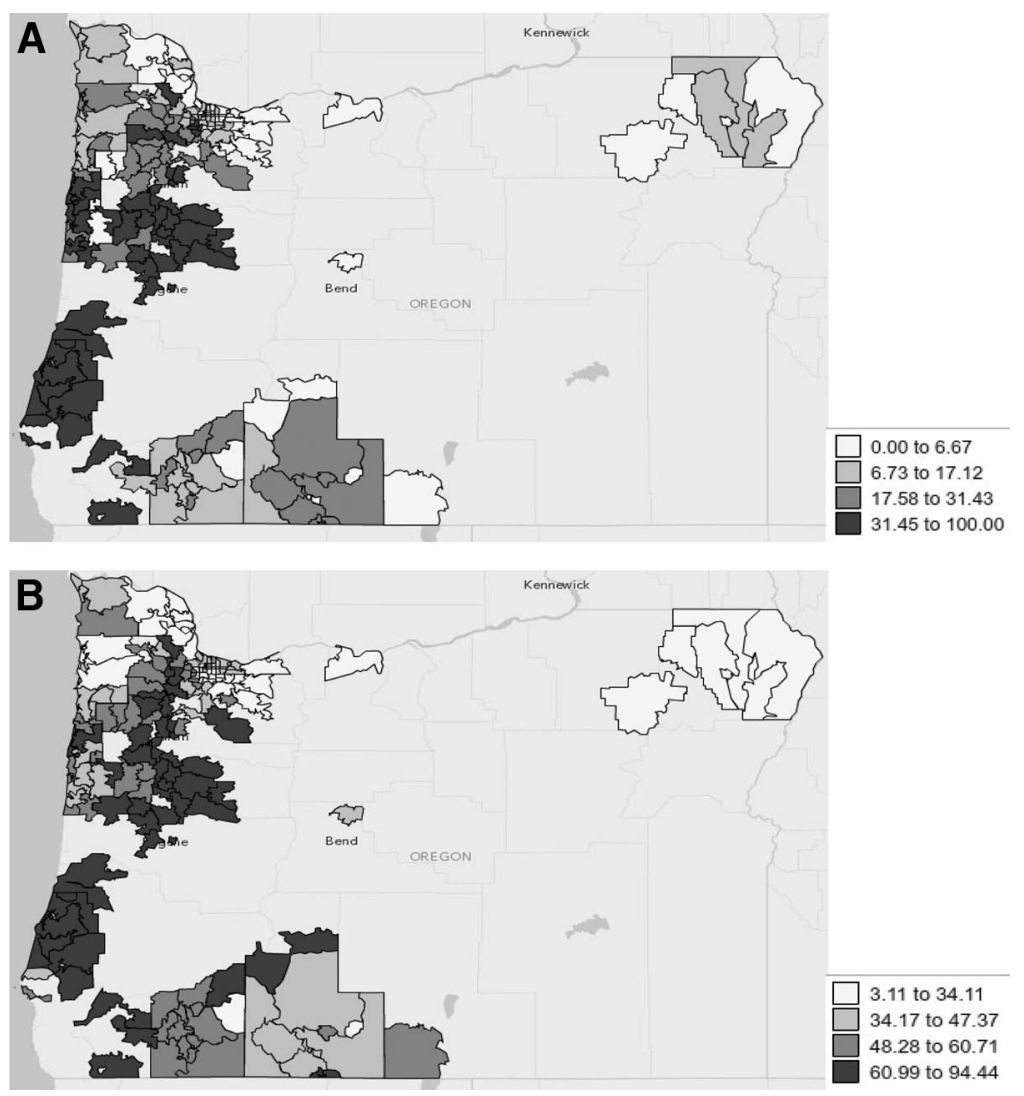

tools available directly to network clinics and how best to work with them to use the tools. GIS tools can be used at the clinic level to investigate clinically relevant questions and visualize patient-level characteristics and determinants of health. This could help clinics to target outreach efforts or community interventions. For example, areas with a high prevalence of chronic disease or low rates of preventive cancer screening can be used to inform the development of a program that sends educators on home visits or forms a partnership among community health workers to focus on needed disease prevention. Because most EHR-based panel management systems have limited ability to display tables or graphs, GIS provides a new, easily understandable way for clinicians, patients, community leaders, and researchers to see where interventions are needed. GIS can also enable public health and primary care professionals to more easily engage with each other and set priorities for partnerships based on the prevalence of conditions among shared populations. These data will also support efforts to identify potential partners in public health, social service, and community agencies to integrate population health improvement.

\section{Limitations}

Use of this geographic visualization of EHR data to conduct insurance surveillance is most relevant for clinics with a large percentage of patients without insurance or patients with sporadic coverage. Clinics that see mostly insured patients may be interested in mapping different EHR variables. GIS mapping can be time consuming, and clinicians have not traditionally been paid for this type of work. In the future, however, new GIS software may improve the feasibility of this type of work, and population-based capitated payment systems may increase both the necessity of and compensation for outreach planning. 
Figure 2. Areas with High Rates of Adults and Children without Insurance in OCHIN and Median Household Income Rates by Oregon County by ZIP code. (A) Percentage of children without insurance at first visit in Lincoln, Benton, Polk, Marion Counties, Oregon: <1-18 years of age with at least one visit to any OCHIN PBRN clinic in 2011 (B) Percentage of adults without insurance at first visit in Lincoln, Benton, Polk, Marion Counties, Oregon: 19-64 years of age with at least one visit to any OCHIN PBRN clinic in 2011. (C) Median Household Income (in dollars) at first visit in Lincoln, Benton, Polk, Marion Counties, Oregon: <1-64 years of age with at least one visit to any OCHIN PBRN clinic in 2011.
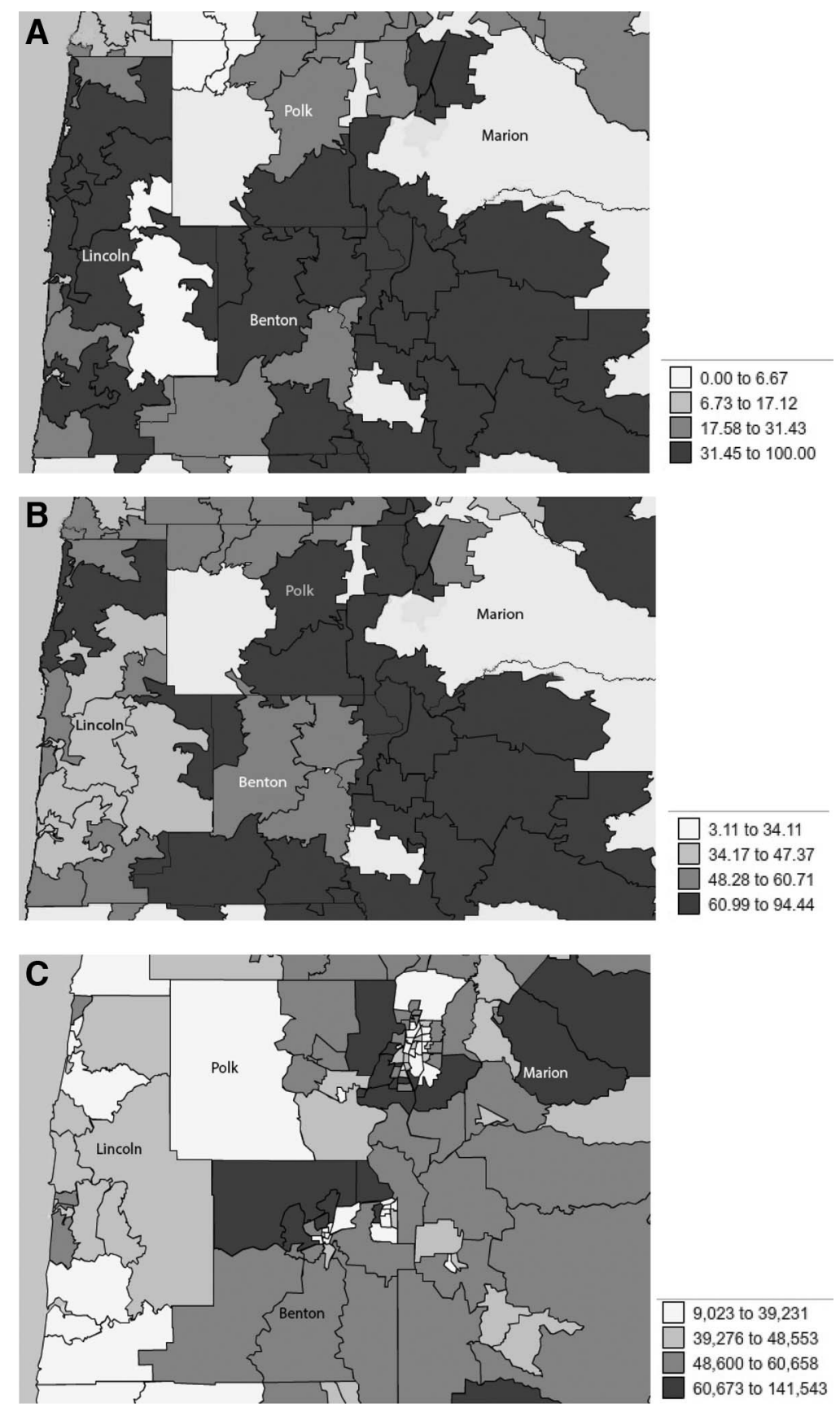

To take full advantage of similar GIS capabilities, PBRNs will need to address multiple technical issues including data aggregation, completeness, and accuracy. For example, there may be instances of incorrect or incomplete ZIP codes for a number of patients, and systems for tracking patients who move often (eg, patients covered by Medicaid) or are seen at $>1$ clinic need to be developed. How- 
Figure 3. Use of Geographic Information Systems (GIS) to identify clinics with patients most likely to benefit from a health insurance outreach intervention. EHR, electronic health record.

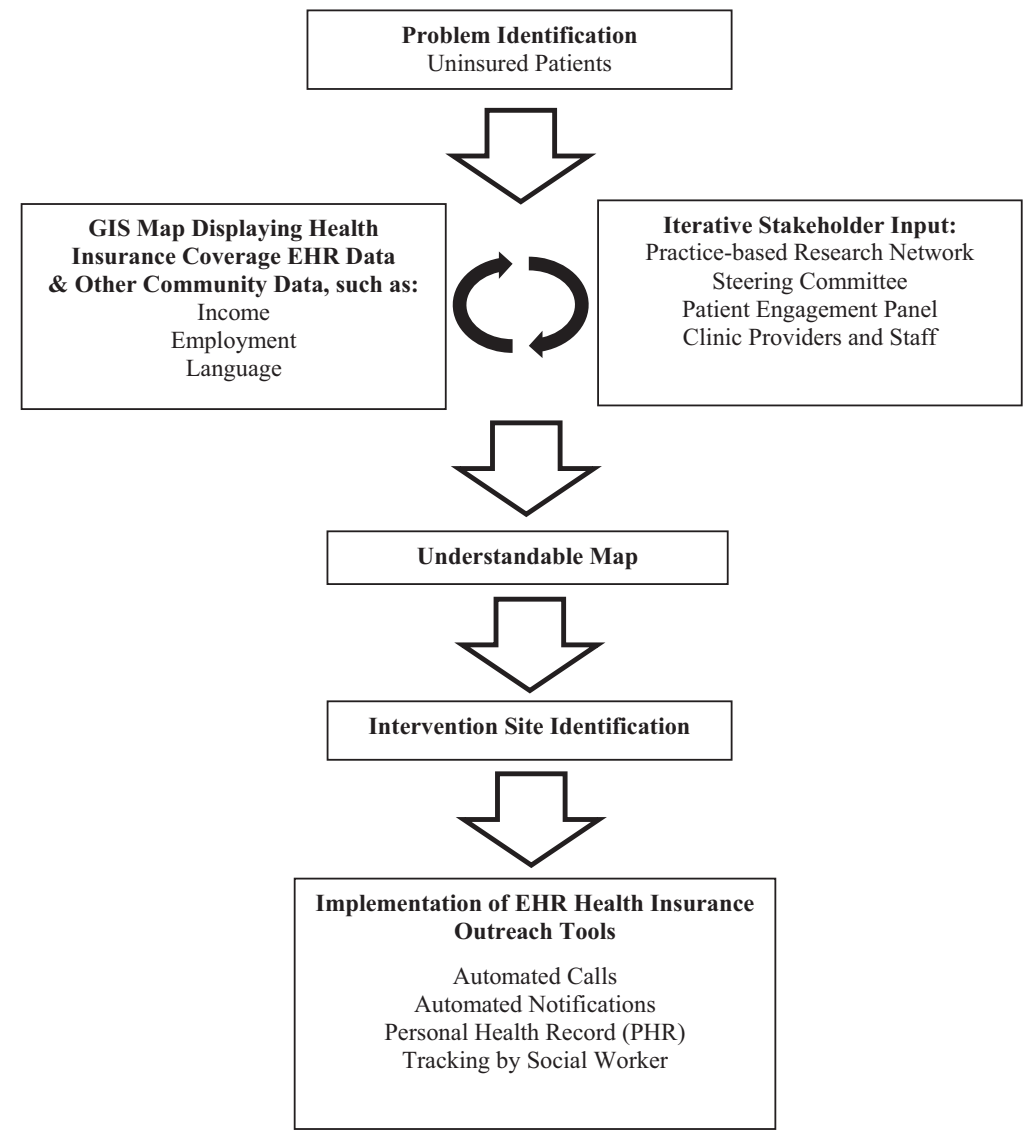

ever, Bailey and colleagues ${ }^{27}$ found that only $10 \%$ of patients in a sample of OCHIN clinics in Oregon had a visit at $>1$ clinic. ZIP codes themselves have limitations; because the geographic areas comprising ZIP codes vary in size, we lose the visualization of some of the small ZIP codes in dense urban areas. However, the ZIP code is the easiest way to use address data without further manipulation (such as a crosswalk to census tract). In addition, the percentages shown on the maps were not standardized; some ZIP codes with a small number of patients may have had a large percentage of uninsured patients. Thus, we did not rely solely on the maps but used the knowledge of our steering committee, PEP, and clinics to make the final determination of where the tools should be implemented.

\section{Conclusion}

The visualization of EHR data using GIS webbased mapping was useful in identifying communi- ties with a large percentage of patients without insurance in the OCHIN PBRN. These findings have directly informed the dissemination of an EHR-based health insurance outreach intervention. Using additional EHR data elements, this method could be generalized for use by PBRNs, primary care clinicians, public health officials, and others to identify a wide array of practice and community needs and to appropriately implement targeted interventions.

The authors acknowledge the OCHIN PBRN Steering Committee and clinics and the Patient Engagement Panel that contributed to this study.

\section{References}

1. Office of the Legislative Council. Compilation of Patient Protection and Affordable Care Act. 111th Congress, 2d session. 2010. Available from: http:// docs.house.gov/energycommerce/ppacacon.pdf. Accessed September 20, 2014. 
2. Sommers BD, Rosenbaum S. Issues in health reform: how changes in eligibility may move millions back and forth between Medicaid and insurance exchanges. Health Aff (Millwood) 2011;230:228-36.

3. Cassedy A, Fairbrother G, Newacheck PW. The impact of insurance instability on children's access, utilization, and satisfaction with health care. Ambul Pediatr. 2008;8:321-8.

4. DeVoe JE, Ray M, Krois L, Carlson MJ. Uncertain health insurance coverage and unmet children's health care needs. Fam Med 2010;42:121-32.

5. Hoffman C, Paradise J. Health insurance and access to health care in the United States. Ann N Y Acad Sci 2008;1136:149-60.

6. Nichols GA, Desai J, Elston Lafata J, et al; SUPREMEDM Study Group. Construction of a multisite DataLink using electronic health records for the identification, surveillance, prevention, and management of diabetes mellitus: the SUPREME-DM project. Prev Chronic Dis 2012;9:E110.

7. Serrano N, Molander R, Monden K, Grosshans A, Krahn DD. Exemplars in the use of technology for management of depression in primary care. WMJ 2012;111:112-8.

8. Hatch B, Angier H, Marino M, et al. Using electronic health records to conduct children's health insurance surveillance. Pediatrics 2013;132:e1584-91.

9. Devoe JE. Being uninsured is bad for your health: can medical homes play a role in treating the uninsurance ailment? Ann Fam Med 2013;11:473-6.

10. DeVoe J, Angier H, Likumahuwa S, et al. Use of qualitative methods and user-centered design to develop customized health information technology tools within federally qualified health centers to keep children insured. J Ambul Care Manag 2014;37: $148-54$.

11. U.S. Department of Health and Human Services. The Affordable Care Act and Health Centers. 2013; Available from: http://www.bphc.hrsa.gov/ about/healthcenterfactsheet.pdf. Accessed October 17, 2014.

12. Miranda ML, Ferranti J, Strauss B, Neelon B, Califf RM. Geographic health information systems: a platform to support the "triple aim." Health Aff (Millwood) 2013;32:1608-15.

13. Bazemore A, Phillips RL, Miyoshi T. Harnessing geographic information systems (GIS) to enable community-oriented primary care. J Am Board Fam Med 2010;23:22-31.

14. Aronson RE, Wallis AB, O'Campo PJ, Schafer P. Neighborhood mapping and evaluation: a methodology for participatory community health initiatives. Matern Child Health J 2007;11:373-83.
15. Dubowitz T, Williams M, Steiner ED, et al. Using geographic information systems to match local health needs with public health services and programs. Am J Public Health 2011;101:1664-5.

16. Auchincloss AH, Gebreab SY, Mair C, Diez Roux AV. A review of spatial methods in epidemiology, 2000-2010. Annu Rev Public Health 2012;33: 107-22.

17. Beyer KM, Rushton G. Mapping cancer for community engagement. Prev Chronic Dis. 2009;6:A03.

18. Castronovo DA, Chui KK, Naumova EN. Dynamic maps: a visual-analytic methodology for exploring spatio-temporal disease patterns. Environ Health 2009;8:61.

19. Dulin MF, Ludden TM, Tapp H, et al. Using geographic information systems (GIS) to understand a community's primary care needs. J Am Board Fam Med 2010;23:13-21.

20. Dulin MF, Ludden TM, Tapp H, et al. Geographic information systems (GIS) demonstrating primary care needs for a transitioning Hispanic community. J Am Board Fam Med 2010;23:109-20.

21. Bazemore A, Diller P, Carrozza M. The impact of a clinic move on vulnerable patients with chronic disease: a geographic information systems (GIS) analysis. J Am Board Fam Med 2010;23:128-30.

22. Taylor DM, Yeager VA, Ouimet C, Menachemi N. Using GIS for administrative decision-making in a local public health setting. Public Health Rep 2012; 127:347-53.

23. Guilbert TW, Arndt B, Temte J, et al. The theory and application of UW ehealth-PHINEX, a clinical electronic health record-public health information exchange. WMJ 2012;111:124-33.

24. DeVoe JE, Gold R, Spofford M, et al. Developing a network of community health centers with a common electronic health record: description of the Safety Net West Practice-based Research Network (SNW-PBRN). J Am Board Fam Med 2011;24:597604.

25. DeVoe J, Sears A. OCHIN Community Information Network: bringing together community health centers, information technology, and data to support a patient-centered medical village. J Am Board Fam Med 2013;26:271-8.

26. Community HealthView. Cincinnati (OH): HealthLandscape; 2013. Available from: www.healthlandscape.org. Accessed September 30, 2013.

27. Bailey S, O'Malley JP, Gold R, Heintzman J, Likumahuwa S, DeVoe JE. Diabetes care quality is highly correlated with patient panel characteristics. J Am Board Fam Med 2013;26:669-79. 